\title{
Gamow-Teller strength distributions at finite temperature and electron capture in stellar environment
}

\author{
A. A. Dzhioev ${ }^{* a} \dagger$ A. I. Vdovin ${ }^{a}$, V. Yu. Ponomarev ${ }^{b}$, J. Wambach ${ }^{b, c}$, \\ K. Langanke ${ }^{b, c, d}$, and G. Martínez-Pinedo ${ }^{c}$ \\ ${ }^{a}$ Bogoliubov Laboratory of Theoretical Physics, JINR, RU-141980 Dubna, Russia \\ ${ }^{b}$ Institut für Kernphysik, Technische Universität Darmstadt, D-64289 Darmstadt, Germany \\ ${ }^{c}$ GSI Helmholtzzentrum für Schwerionenforschung, D-64291 Darmstadt, Germany \\ ${ }^{d}$ Frankfurt Institute for Advanced Studies, D-60438 Frankfurt, Germany
}

E-mail: dzhioevdtheor.jinr.ru

The thermal evolutions of the $\mathrm{GT}_{+}$distributions are presented for the sample nuclei ${ }^{54} \mathrm{Fe}$ and ${ }^{76} \mathrm{Ge}$. They are obtained within the proton-neutron QRPA extended to finite temperature by the thermofield dynamics formalism. The strength distributions are used to calculate stellar electron capture rates and cross sections.

PACS: $26.50 .+x, 23.40 .-s, 21.60 . \mathrm{Jz}, 24.10 . \mathrm{Pa}$

11th Symposium on Nuclei in the Cosmos, NIC XI

July 19-23, 2010

Heidelberg, Germany

\footnotetext{
* Speaker.

${ }^{\dagger}$ This work was supported in part by HIC for Fair, the Helmholtz Alliance EMMI, the Heisenberg-Landau Program, and by the DFG grant (SFB 634).
} 


\section{Introduction}

It is well known that reliable electron capture rates are essential for simulations of corecollapse supernovae and their calculation requires a detailed knowledge of the Gamow-Teller strength distributions in nuclei [四]. Furthermore, the finite temperature in the star requires the implicit consideration of capture on excited nuclear states, for which the $\mathrm{GT}_{+}$distribution can be very different from the one for the ground states.

To date, the most reliable calculations of electron capture rates have been performed for iron group nuclei using a large-scale shell model (LSSM) diagonalization approach [D]. For more massive and neutron-rich nuclei electron capture rates have been derived within the framework of a hybrid model combining the Shell Model Monte-Carlo (SMMC) approach and the Random Phase Approximation (RPA) [䧃]. Nevertheless, both approaches have their own shortcomings: The LSSM partially employs the Brink hypothesis when treating GT transitions from nuclear excited states. The hybrid model is free of this disadvantage, but it does not include explicitly pairing correlations when calculating the strength distributions. An alternative method for description of Gamow-Teller strength distributions and electron capture rates at finite temperatures is presented in this talk.

\section{Approach}

In Ref. [[]], we have introduced a theory based on the proton-neutron QRPA extended to finite temperature (TQRPA) by the thermofield dynamics formalism (TFD) [ 6$]$. This technique does not rely on Brink's hypothesis and allows to calculate the strength and the energies of allowed and forbidden transitions as functions of the nuclear temperature. Moreover, the Ikeda sum rule for Fermi and GT transitions is fulfilled within the approach. In our study we use a phenomenological nuclear Hamiltonian of the quasiparticle-phonon nuclear model (QPM) [U]. It consists of a spherically symmetric Woods-Saxon mean field potential for protons and neutrons, BCS pairing interactions and separable multipole and spin-multipole particle-hole interactions. All parameters in the QPM Hamiltonian are fitted to reproduce experimental data at zero temperature (see Ref. [G] for more details).

\section{The results}

The temperature evolution of the $\mathrm{GT}_{+}$strength distribution for ${ }^{54} \mathrm{Fe}$ is displayed in Fig. $\mathrm{W}(\mathrm{lhs})$. With increasing temperature two effects occur in our model that influence the $\mathrm{GT}_{+}$strength distribution: (i) At low temperatures, due to pairing, $\mathrm{GT}_{+}$transitions involve the breaking of a proton Cooper pair associated with some energy cost. This extra energy vanishes at temperatures higher then critical one ( $T_{\mathrm{cr}} \approx 0.8 \mathrm{MeV}$ ). (ii) $\mathrm{GT}_{+}$transitions, which are Pauli blocked at low temperatures due to closed neutron subshells (e.g., $1 f_{7 / 2}$ orbital), become thermally unblocked as temperature increases. Similarly, protons which are thermally excited to higher orbitals can undergo GT + transitions. Because of thermally unblocked transitions, some $\mathrm{GT}_{+}$strength appears well below the zero-temperature threshold, including negative energies.

Due to the vanishing of the pairing correlations and appearance of negative- and low-energy transitions, the centroid of the $\mathrm{GT}_{+}$strength distribution in ${ }^{54} \mathrm{Fe}$ is shifted to lower excitation energies at high temperatures. Our calculations indicate that as temperature increases to $0.8 \mathrm{MeV}$, 

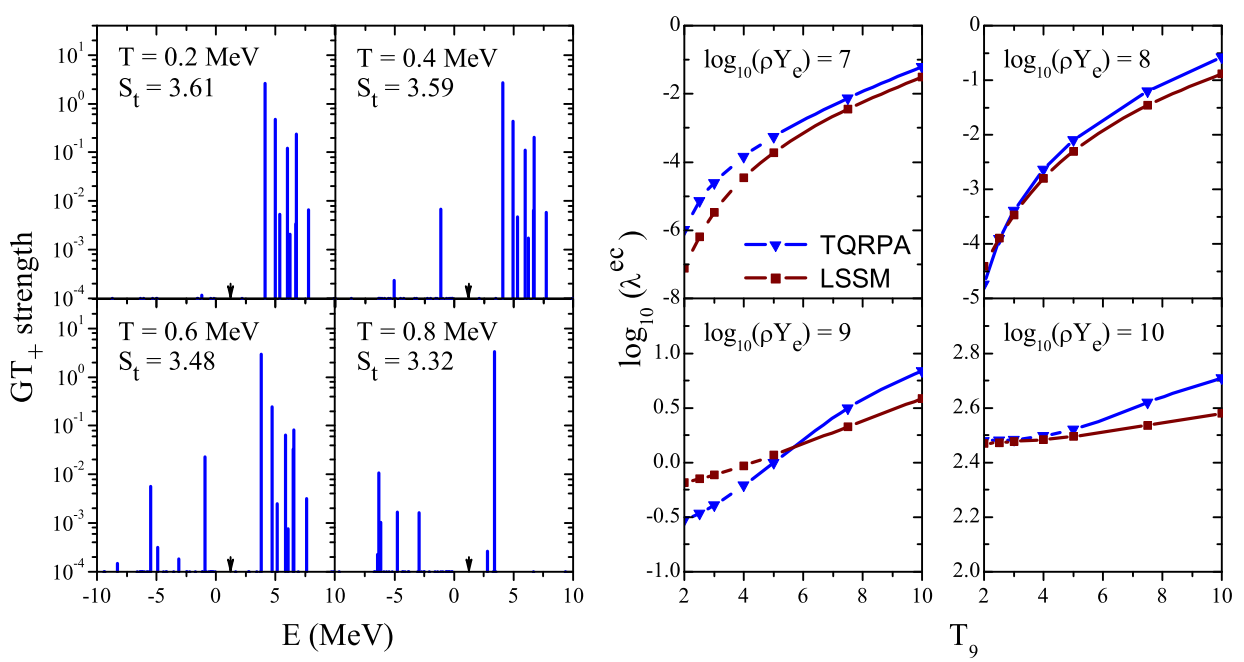

Figure 1: (Left panel) Temperature evolution of $\mathrm{GT}_{+}$strength distributions for ${ }^{54} \mathrm{Fe}$ versus parent excitation energy. $S_{t}$ is the total $\mathrm{GT}_{+}$strength. The arrows indicate the zero-temperature threshold $Q=M_{f}-M_{i}=$ $1.21 \mathrm{MeV}$, where $M_{i, f}$ are the masses of the parent and daughter nuclei. (Right panel) Electron capture rates for ${ }^{54} \mathrm{Fe}$ calculated using the TQRPA and LSSM approaches as a function of temperature $\left(T_{9}=10^{9} \mathrm{~K}\right)$ for selected values of density $\rho Y_{e}$ (in $\mathrm{g} \mathrm{cm}^{-3}$ ).

the $\mathrm{GT}_{+}$centroid shifts by $1.5 \mathrm{MeV}$. Thus, the present approach does not support Brink's hypothesis. Similar results have been obtained in SMMC calculations of the GT centroids at finite temperatures [ [8]. A gradual decrease of the total $\mathrm{GT}_{+}$strength $S_{t}$ takes place when the temperature increases from zero to $0.8 \mathrm{MeV}$ (see $S_{t}$ values in Fig. (口(lhs)).

In Fig. W(rhs), we compare electron capture rates calculated in the TQRPA with the ones calculated using the LSSM approach [B]. There is a certain disagreement between them. At low temperatures $(T)$ and densities $(\rho)$ the disagreement is due to difference in the strength and the energy of negative-energy transitions which dominate the rates at low $(T, \rho)$. At low $T$ and intermediate $\rho$, the near threshold part of the $\mathrm{GT}_{+}$strength dominates the capture rates. The TQRPA accounts for the Landau damping only and thus, underestimates fragmentation of the $\mathrm{GT}_{+}$strength as compared to the LSSM, leading to too small strength at the threshold. Accordingly our rates appear to be smaller than the LSSM ones. Accounting for coupling of one-phonon states to complex configurations in our model should make predictions of two approaches closer. At high temperatures the rates are always dominated by strong transitions involving the $\mathrm{GT}_{+}$resonance. Since the present approach predicts that with increasing temperatures the $\mathrm{GT}_{+}$resonance moves to lower excitation energies, our high temperature rates always slightly surpass the shell-model ones.

The strength distribution of $\mathrm{GT}_{+}$transitions from the even germanium isotope ${ }^{76} \mathrm{Ge}$ is displayed in Fig. $\mathbb{\nabla}(\mathrm{lhs})$. The distribution has been folded with a Breit-Wigner function of $1 \mathrm{MeV}$ width. With increasing temperature, the peaks in the $\mathrm{GT}_{+}$distribution shift to lower excitation energies and the total strength decreases in the vicinity of the critical temperature $\left(T_{\mathrm{cr}} \sim 0.9 \mathrm{MeV}\right)$. The shift is about $8 \mathrm{MeV}$ and, hence, cannot be explained solely by disappearing of the extra energy needed to break a proton pair. To explain both the effects we consider single-particle transitions which mainly contribute to the total $\mathrm{GT}_{+}$strength in ${ }^{76} \mathrm{Ge}$. These are the $1 g_{9 / 2}^{p} \rightarrow 1 g_{7 / 2}^{n}$ particle- 

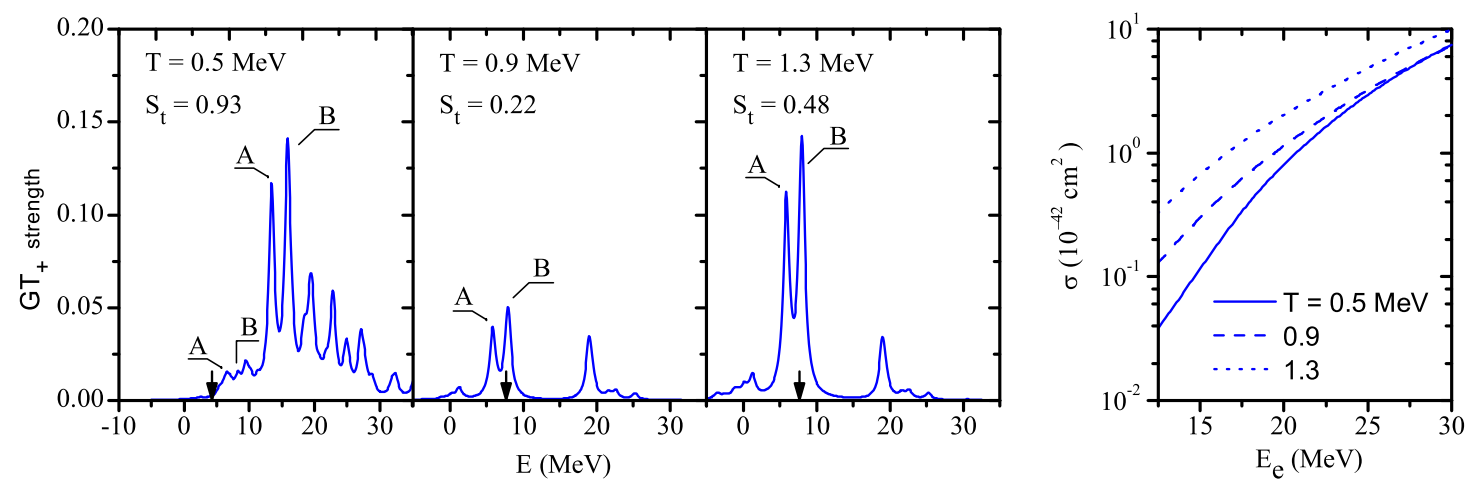

Figure 2: (Left panel) Strength distribution (folded) of $\mathrm{GT}_{+}$transitions in ${ }^{76} \mathrm{Ge}$ at various temperatures. The arrows indicate the zero-temperature threshold $Q=7.52 \mathrm{MeV}$. $A$ and $B$ label the transitions: $A \equiv 1 f_{7 / 2}^{p} \rightarrow$ $1 f_{5 / 2}^{n}, B \equiv 1 g_{9 / 2}^{p} \rightarrow 1 g_{7 / 2}^{n}$. (Right panel) Electron capture cross sections for ${ }^{76} \mathrm{Ge}$ calculated within the TQRPA approach for various temperatures. $E_{e}$ is the electron energy.

particle and $1 f_{7 / 2}^{p} \rightarrow 1 f_{5 / 2}^{n}$ hole-hole transitions. Both transitions are blocked at zero temperature in an independent particle model. At relatively low temperatures, configuration mixing induced by pairing correlations in the ground state is the main unblocking mechanism. The position of the $\mathrm{GT}_{+}$peaks is given by $\varepsilon_{1 g_{9 / 2}}^{p}+\varepsilon_{1 g_{7 / 2}}^{n}+\delta_{n p}$ and $\varepsilon_{1 f_{7 / 2}}^{p}+\varepsilon_{1 f_{5 / 2}}^{n}+\delta_{n p}$, where $\varepsilon_{j}^{p(n)}$ is the proton (neutron) quasiparticle energy. The quantity $\delta_{n p}=\Delta \mu_{n p}+\Delta M_{n p}$ takes into account the difference between the proton and the neutron chemical potentials and the proton-neutron mass splitting. As a result, at low temperatures the $\mathrm{GT}_{+}$peaks resides at energies around $15 \mathrm{MeV}$. When pairing correlations disappear at temperatures near the critical one, the corresponding peaks completely vanish. The total $\mathrm{GT}_{+}$strength is noticeably reduced. At higher temperatures, the above mentioned single transitions become unblocked due to thermal excitations [Q]. But now their energies are determined by the difference of quasiparticle energies, $\varepsilon_{1 g_{7 / 2}}^{n}-\varepsilon_{1 g_{9 / 2}}^{p}+\delta_{n p}$ and $\varepsilon_{1 f_{7 / 2}}^{p}-\varepsilon_{1 f_{5 / 2}}^{n}+\delta_{n p}$. As a result the $\mathrm{GT}_{+}$peaks appear near the zero-temperature threshold. Obviously, the significant shift of the $\mathrm{GT}_{+}$peaks to lower excitation energies favors electron capture.

No shift to lower excitation energies was observed within the hybrid model calculations [四]. In the hybrid model, occupation numbers at finite temperature are calculated within the SMMC approach, accounting for all many-body $n \mathrm{p} n \mathrm{~h}$ correlations induced by a pairing+quadrupole residual interaction. These occupation numbers have been then used to define a thermal ground state that is the basis of an RPA approach to calculate the electron capture cross sections, considering only $1 \mathrm{p} 1 \mathrm{~h}$ excitations on the top this ground state. Therefore the hybrid model does not include explicitly $2 \mathrm{p} 2 \mathrm{~h}$ pairing correlations when calculating strength distributions. As a consequence the energy of $\mathrm{GT}_{+}$transitions is nearly independent on the temperature within the hybrid model and $\mathrm{GT}_{+}$peaks are always located near the zero-temperature threshold.

To reveal the importance of the thermal unblocking for $\mathrm{GT}_{+}$transitions in neutron-rich nuclei we perform electron capture cross section calculations (see Fig. $\mathbb{\nabla}($ rhs)) taking into account the contribution of $\mathrm{GT}_{+}$and first-forbidden transitions in our calculations. The strong temperature sensitivity of the cross section at low electron energies reflects the temperature dependence of the TQRPA GT + strength distribution in our model. It is amplified by the strong phase space 
energy dependence, leading to a much stronger temperature dependence of the cross section in the TQRPA model than in the hybrid model [䧃]. For higher electron energies, the first-forbidden transitions become increasingly important [Q] . As the strength of the first-forbidden transitions is less sensitive to temperature [罒, the capture cross sections at $E_{e} \sim 30 \mathrm{MeV}$ depend only weakly on temperature.

\section{Conclusion and outlook.}

We have presented the approach that allows calculations of stellar weak-interaction processes at finite temperature in a thermodynamically consistent way. It accounts for correlations described by the TQRPA. Our approach is conceptually superior to the hybrid approach of the SMMC+RPA which has previously been used to estimate electron capture rates for neutron-rich nuclei. Whereas much of the essential physics is already recovered, the detailed comparison to the shell model results implies that the approach should be further improved. The improvement would be inclusion of correlations beyond TQRPA by coupling one-phonon states to more complex (e.g., two-phonon)

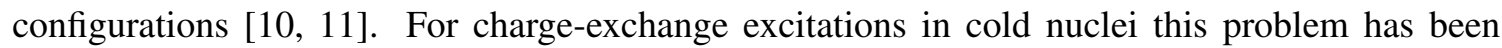
considered within the QPM [12] and by approaches that solve the (second) RPA equations in the space of two-particle/two-hole excitations [[13]]. Another direction is the combination of our TFD based approach at finite temperature with self-consistent QRPA calculations based on more realistic effective interaction [1]4].

\section{References}

[1] K. Langanke and G. Martínez-Pinedo, Rev. Mod. Phys. 75 (2003) 819.

[2] E. Caurier, K. Langanke, G. Martínez-Pinedo, and E. Nowacki, Nucl. Phys. A 653 (1999) 439; K. Langanke and G. Martínez-Pinedo, Nucl. Phys. A 673 (2000) 481.

[3] K. Langanke and G. Martínez-Pinedo, At. Data Nucl. Data Tables 79 (2001) 1.

[4] K. Langanke, E. Kolbe, and D. J. Dean, Phys. Rev. C 63 (2001) 032801(R).

[5] A. A. Dzhioev, et al., Phys. Rev. C 81 (2010) 015804.

[6] Y. Takahashi and H. Umezawa, Collect. Phenom. 2 (1975) 55.

[7] V. G. Soloviev, Theory of atomic nuclei: quasiparticles and phonons, Institute of Physics Publishing, Bristol and Philadelphia, 1992.

[8] P. B. Radha, et al., Phys. Rev. C 56 (1997) 3079.

[9] J. Cooperstaine and J. Wambach, Nucl. Phys. A 420 (1984) 591.

[10] A. A. Dzhioev and A. I. Vdovin, Int. J. Mod. Phys. E 18 (2009) 1535.

[11] A. A. Dzhioev and A. I. Vdovin, Phys. of Part. and Nucl. 41 (2010) 1127.

[12] V. A. Kuzmin and V. G. Soloviev, J. Phys. G 10 (1984) 1507; V. A. Kuzmin and V. G. Soloviev, J. Phys. G 11 (1985) 603.

[13] S. Drożdż, V. Klemt, J. Speth, and J. Wambach, Phys. Lett. B 166 (1986) 18; S. Drożdż, F. Osterfeld, J. Speth, and J. Wambach, Phys. Lett. B 189 (1987) 271.

[14] J. Engel, et al., Phys. Rev. C 60 (1999) 014302. 